\title{
Medidas de distanciamento social para o enfrentamento da COVID-19 no Brasil: caracterização e análise epidemiológica por estado
}

\section{Social distancing measures in the fight against COVID-19 in Brazil: description and epidemiological analysis by state}

\section{Medidas de distanciamiento social para el combate a la COVID-19 en Brasil: caracterización y análisis epidemiológico por estado}

Lara Livia Santos da Silva 1

Alex Felipe Rodrigues Lima 2

Démerson André Polli 3

Paulo Fellipe Silvério Razia 1

Luis Felipe Alvim Pavão 4

Marco Antônio Freitas de Hollanda Cavalcanti 5 Cristiana Maria Toscano 1

doi: 10.1590/0102-311X00185020

\section{Resumo}

Medidas de distanciamento social vêm sendo amplamente adotadas para mitigar a pandemia da COVID-19. No entanto, pouco se sabe quanto ao seu impacto no momento da implementação, abrangência e duração da vigência das medidas. $O$ objetivo deste estudo foi caracterizar as medidas de distanciamento social implementadas pelas Unidades da Federação (UF) brasileiras, incluindo o tipo de medida e o momento de sua adoção. Trata-se de um estudo descritivo com caracterização do tipo, momento cronológico e epidemiológico da implementação e abrangência das medidas. O levantamento das medidas foi realizado por meio de buscas em sites oficiais das Secretarias de Governo e no Diário Oficial de cada UF. Os números de casos e óbitos por COVID-19 foram obtidos de uma plataforma de informações oficiais. Consideramos as seguintes categorias de medidas de distanciamento social: suspensão de eventos, suspensão de aulas, quarentena para grupos de risco, paralisação econômica (parcial ou plena), restrição de transporte e quarentena para a população. $O$ momento de implementação considerou a data cronológica e também o momento epidemiológico, levando em conta o tempo após o décimo caso ou primeiro óbito por COVID-19 em cada UF. Todas as UF implementaram medidas de distanciamento, em sua maioria durante a segunda quinzena de março de 2020. Paralisação econômica foi implementada precocemente, anterior ao décimo caso por $67 \%$ e anterior ao primeiro óbito por COVID-19 por $89 \%$ das UF. As medidas de distanciamento social foram amplamente implementadas no Brasil, de maneira precoce, antes ou na fase inicial da curva de crescimento exponencial de casos ébitos por COVID-19 na grande maioria das UF.

\section{Correspondência}

L. L. S. Silva

Universidade Federal de Goiás.

Rua 235 s/n, Setor Leste Universitário, Goiânia, GO 74605-050, Brasil.

laraliviasantos@gmail.com

1 Universidade Federal de Goiás, Goiânia, Brasil. 2 Instituto Mauro Borges de Estatística e Estudos Socioeconômicos, Goiânia, Brasil.

3 Universidade de Brasília, Brasília, Brasil.

4 Secretaria do Tesouro Nacional, Brasília, Brasil.

5 Instituto de Pesquisa Econômica Aplicada,

Rio de Janeiro, Brasil. 


\section{Introdução}

Em 11 de março de 2020, a Organização Mundial da Saúde (OMS) declarou a COVID-19, doença causada pelo novo coronavírus denominado SARS-CoV-2 (síndrome respiratória aguda grave coronavírus 2), como pandemia. Essa declaração ocorreu no momento em que a epidemia, iniciada em Wuhan, na China, já estava presente em 114 países/territórios/áreas, alcançando a marca de 118.319 casos e 4.292 óbitos pela doença 1. Três meses depois, já havia a notificação de mais de sete milhões de pessoas no mundo com a doença e mais de 408 mil óbitos decorrentes da COVID-19, ocorridos em 215 países/territórios/áreas ao redor do mundo 2.

A rápida disseminação do SARS-CoV-2 entre países e comunidades, resultante da alta transmissibilidade viral, associada à inexistência de vacinas e antivirais específicos eficazes para a prevenção e tratamento da doença, torna as intervenções não farmacológicas as opções mais eficientes para a mitigação e controle da COVID-19 em nível local e global 3,4. Dentre essas intervenções, em nível populacional, há as medidas de distanciamento social, cujo termo se refere a esforços que visam a diminuir ou interromper a cadeia de transmissão da doença pelo distanciamento físico entre indivíduos que possam estar infectados e os saudáveis, além de proteger aqueles indivíduos em risco de desenvolver a forma grave da doença. Incluem-se nessas medidas o cancelamento de eventos em massa, fechamento temporário de escolas e locais de trabalho, bloqueio de fronteiras e a recomendação para a população ficar em casa 5 .

As medidas de distanciamento social já foram utilizadas na mitigação de epidemias e pandemias anteriores, incluindo a pandemia de influenza de 1918-1919 6, a epidemia de síndrome respiratória aguda grave (SARS) em 2002-2003 7 e a pandemia de influenza H1N1 em 2009-2010 8, sendo, na atualidade, amplamente recomendadas para a mitigação e combate à pandemia do COVID-19 5,9,10,11.

A começar por Wuhan, que iniciou a implementação das medidas de distanciamento social aproximadamente três semanas após o início da epidemia 12, outras cidades chinesas, países asiáticos, bem como diversos países ao redor do mundo adotaram, sucessivamente, medidas de distanciamento social à proporção que a transmissão comunitária foi se confirmando nesses locais 13. Evidências iniciais já apontam que a adoção dessa estratégia em diversos cenários tem sido efetiva para conter o avanço descontrolado de casos e óbitos pela COVID-19, especialmente quando associada ao isolamento e à quarentena dos contatos 11,12,14,15,16,17. No entanto, ainda há incerteza sobre a efetividade dos diferentes tipos de medidas quando consideradas individualmente ou de maneira combinada, bem como sobre o efeito do momento da implementação dessas medidas na progressão epidemiológica da doença.

Para além do setor saúde, é importante considerar os inevitáveis impactos sociais e econômicos que a implementação das medidas de distanciamento social irá necessariamente causar 18. Por esse motivo, é importante que haja uma avaliação cuidadosa do momento epidemiológico mais adequado para a aplicação dessas medidas, assim como a sua vigência, com o objetivo de maximizar os efeitos desejáveis na saúde enquanto minimiza os danos sociais e econômicos 3 .

No Brasil, o primeiro caso de COVID-19 foi confirmado em 26 de fevereiro de 2020 na cidade de São Paulo ${ }^{19}$. Após um mês dessa primeira confirmação, todos os estados brasileiros já haviam notificado casos da doença, com mortes registradas em oito deles 20 . A evolução observada do número de casos e óbitos nas Unidades da Federação (UF) brasileiras é, no entanto, distinta 21, possivelmente em função de diferenças sociodemográficas e geográficas, do momento da introdução da infecção e também por conta da autonomia que os estados e municípios têm de definir quais e quando cada medida de mitigação da COVID-19 será implementada em seu cenário 22. É necessário, portanto, o mapeamento das medidas adotadas nas diversas UF a fim de se levantarem hipóteses e de se avaliar o momento mais adequado de implementação e o resultado de diferentes medidas em diferentes UF e regiões brasileiras. Para responder a essas questões, é fundamental dispor de uma base de dados completa obtida a partir de um processo sistemático de busca, que registre os tipos de medidas adotadas em cada UF, juntamente com os momentos de sua implementação.

Neste sentido, o objetivo deste estudo foi caracterizar as medidas de distanciamento social implementadas pelas UF brasileiras, incluindo o tipo de medida e o momento cronológico e epidemiológico de sua implementação, considerando o número de casos e óbitos por COVID-19 em cada local. 


\section{Métodos}

\section{Delineamento, local e período do estudo}

Trata-se de um estudo descritivo realizado com dados de cada uma das 27 UF brasileiras, no período compreendido entre a ocorrência do primeiro caso confirmado de COVID-19 no país, dia 26 de fevereiro de 2020, até o dia 30 de junho de 2020.

\section{Fonte e coleta de dados}

\section{- Medidas de distanciamento social}

O levantamento das legislações referentes à implementação das medidas de distanciamento social foi realizado para cada UF brasileira. A busca foi realizada nas páginas eletrônicas oficiais do Governo e no Diário Oficial das UF. Foram considerados decretos, leis, portarias, notas técnicas e deliberações do Comitê Extraordinário COVID-19 (esta última para Minas Gerais), todos publicados no período do estudo.

As seguintes informações foram extraídas das legislações identificadas e inseridas em uma planiTha de dados estruturada para essa finalidade: data da publicação e descrição da norma, tipo de medida implementada e data da implementação. Os dados foram coletados no período de 10 de março de 2020 a 1o de julho de 2020 por uma equipe de três pesquisadores.

\section{- Dados epidemiológicos}

Os números de casos confirmados e óbitos por COVID-19 notificados aos sistemas de vigilância epidemiológica de cada UF de acordo com a padronização nacional e estadual 23, ocorridos durante o período do estudo em cada UF, foram obtidos por meio da plataforma Brasil.io (https://brasil.io/ covid19/), que coleta e revisa diariamente os dados publicados, tornando-os mais acurados e atualizados. Esse site utiliza como fonte os dados oficiais reportados pelos boletins e informes epidemiológicos das Secretarias Estaduais de Saúde das 27 UF 24.

\section{Categorias de medidas de distanciamento social consideradas}

As medidas de distanciamento social foram agrupadas em 10 categorias que foram posteriormente reagrupadas em seis para esta análise, conforme detalhadas no Quadro 1.

\section{Caracterização do momento de implementação das medidas de distanciamento social}

O momento de implementação de cada uma das medidas de distanciamento social considerou a data cronológica e o momento epidemiológico da sua implementação. De maneira geral, para a definição da data cronológica, foram considerados os seguintes critérios: (a) data em que a legislação ou a medida entrou em vigor ou a data em que a legislação indica que a medida deveria ter sido iniciada, e não a data da assinatura da legislação; (b) quando a adoção das medidas ocorreu de maneira progressiva por localidade, como, por exemplo, iniciando na capital e depois sendo implementada na região metropolitana e finalmente em todo o estado, foi considerada a data em que a medida foi implementada em toda a UF; e (c) para todas as categorias, exceto paralisação econômica, considerou-se apenas quando as medidas foram implementadas para todo o estado (e não quando foram implementadas em apenas um ou mais municípios).

Para a categoria paralisação econômica, considerou-se paralisação econômica plena quando todas as cinco medidas de distanciamento foram implementadas, a saber: (1) suspensão do atendimento ao público nos serviços públicos não essenciais e/ou orientação para teletrabalho dos servidores; (2) fechamento de centros comerciais, academias ou centros de esportes privados; (3) suspensão do funcionamento de estabelecimentos alimentícios incluindo proibição de atendimento ao público presencial e/ou consumo no local; (4) suspensão do atendimento ao público de prestadores de servi- 
Quadro 1

Categorias de medidas de distanciamento social e detalhamento das ações consideradas em cada categoria.

\begin{tabular}{|c|c|}
\hline Categorias & Detalhamento \\
\hline Suspensão de eventos & $\begin{array}{l}\text { Suspensão de eventos públicos e/ou privados e atividades culturais, de } \\
\text { lazer, religiosos e esportivos }\end{array}$ \\
\hline Suspensão de aulas & $\begin{array}{l}\text { Suspensão de atividades educacionais (públicas e privadas, incluindo } \\
\text { creche, ensino infantil, fundamental, médio e superior) }\end{array}$ \\
\hline Quarentena de grupos de risco & $\begin{array}{l}\text { Regime especial de trabalho (teletrabalho/home office/trabalho remoto) } \\
\text { para funcionários que estão nos grupos de risco para a COVID-19, a saber: } \\
\text { indivíduos com idade igual ou superior a } 60 \text { anos, gestantes ou lactantes } \\
\text { e/ou portadores de doenças crônicas }\end{array}$ \\
\hline \multirow[t]{5}{*}{$\begin{array}{l}\text { Paralisação econômica } \\
\text { (plena ou parcial) }\end{array}$} & $\begin{array}{l}\text { Suspensão do atendimento ao público nos serviços públicos não essenciais } \\
\text { e/ou orientação para teletrabalho dos servidores }\end{array}$ \\
\hline & $\begin{array}{l}\text { Fechamento de centros comerciais (shoppings, galerias e congêneres), } \\
\text { academias ou centros de esportes privados (ou seja, estabelecimentos } \\
\text { comerciais que geram aglomerações por sua natureza) }\end{array}$ \\
\hline & $\begin{array}{l}\text { Suspensão do funcionamento de estabelecimentos alimentícios (bares, } \\
\text { restaurantes, lojas de conveniência, e similares), incluindo proibição de } \\
\text { atendimento ao público presencial e/ou consumo no local }\end{array}$ \\
\hline & $\begin{array}{l}\text { Suspensão do atendimento ao público de prestadores de serviços e de } \\
\text { demais estabelecimentos comerciais não essenciais }\end{array}$ \\
\hline & Suspensão de atividades industriais não essenciais \\
\hline Restrição do transporte & $\begin{array}{l}\text { Suspensão ou restrição da circulação do transporte de passageiros } \\
\text { intermunicipal e/ou interestadual de qualquer modalidade }\end{array}$ \\
\hline Quarentena da população & Recomendação de quarentena para toda a população \\
\hline
\end{tabular}

ços e de demais estabelecimentos comerciais não essenciais; e (5) suspensão de atividades industriais não essenciais. Por outro lado, considerou-se paralisação econômica parcial quando (1) não houve a implementação de todas as cinco medidas acima ou quando qualquer dessas medidas foi implementada parcialmente (por exemplo, estabelecimento alimentícios ou comerciais com restrição de lotação ou fechamento em apenas algumas circunstâncias); (2) as medidas foram implementadas apenas nas capitais dos estados ou em um grupo de municípios mas não em todo o estado; ou (3) a legislação recomendou, mas não determinou as suspensões.

Especificamente para a categoria suspensão de eventos, foi considerada a data da suspensão de eventos de qualquer natureza, independentemente do número de pessoas, que exigem ou não licença do setor público, assim como a suspensão das atividades culturais, religiosos, esportiva e de lazer. Não foram considerados decretos específicos relacionados a eventos realizados pela administração pública estadual em data anterior. Para a suspensão de aulas, considerou-se a data da suspensão das aulas presenciais da rede pública de ensino. Para a categoria quarentena de grupos de risco, foi considerada a data em que os servidores públicos pertencentes a esses grupos (indivíduos acima de 60 anos, gestantes e/ou portadores de doenças crônicas) foram orientados a laborar fora das instalações físicas do órgão de lotação, em regime de teletrabalho. Para a categoria restrição do transporte, foi considerada a primeira data em que foi determinada a suspensão/proibição do transporte coletivo de passageiros intermunicipal e/ou interestadual, de qualquer modalidade (com exceção do transporte aéreo), público e/ou particular. Também foram considerados os casos em que a suspensão/ proibição do transporte se aplicava somente àqueles oriundos de estados e/ou municípios com casos de COVID-19 ou que declararam situação de emergência decretada. Finalmente, para a categoria 
quarentena da população, considerou-se a data que recomendava à população, a partir daquele momento, limitar seus deslocamentos às atividades estritamente necessárias e/ou aumentar o período de permanência em casa.

O momento epidemiológico da implementação de cada medida foi categorizado, de forma arbitrária, em: (a) precoce, quando ocorreu antes da notificação do primeiro caso de COVID-19; (b) intermediário I, quando ocorreu entre o primeiro e o décimo caso; (c) intermediário II, quando ocorreu entre o décimo primeiro e o quinquagésimo caso; e (d) tardio, quando ocorreu após o quinquagésimo caso. Esse momento foi também apresentado segundo o número de dias que antecederam ou sucederam a data da adoção de cada categoria de medidas a partir do primeiro óbito confirmado da doença.

\section{Síntese e análise dos dados}

Para cada UF foram identificadas as datas cronológicas da implementação de cada medida de distanciamento social e foi calculada a diferença em dias entre a data da adoção da primeira medida e a data da paralisação econômica (plena ou parcial).

O momento epidemiológico da implementação das medidas em cada UF foi categorizado em precoce, intermediário I, intermediário II e tardio. Para a categoria paralisação econômica, esse momento foi apresentado graficamente, a partir do primeiro caso da doença notificado em cada local, em escala logarítmica, agrupado por UF que haviam implementado essa categoria de maneira plena ou parcial. O momento epidemiológico da implementação de cada categoria de medida foi também apresentado segundo o número de dias que antecederam ou sucederam a notificação do primeiro óbito por COVID-19 por UF.

Para a visualização dos dados, foi elaborada uma interface gráfica (https://medidas-covidbr-iptsp. shinyapps.io/painel/) de acesso público que apresenta a evolução dos casos confirmados e óbitos por COVID-19 ao longo do tempo e as medidas de distanciamento social adotadas em cada UF em resposta à pandemia de COVID- 19.

Por se tratar da utilização de dados secundários e de acesso livre, não foi necessária a aprovação do estudo no Comitê de Ética em Pesquisa (CEP).

\section{Resultados}

Em todas as UF brasileiras, foram implementadas quatro ou mais categorias de medidas de distanciamento social. A suspensão de eventos e/ou a quarentena dos grupos de risco foram as primeiras medidas a serem adotadas, com exceção do Tocantins que implementou primeiro a suspensão das aulas. Mato Grosso do Sul, Tocantins, Espírito Santo, São Paulo, Distrito Federal e Rio Grande do Norte não restringiram o transporte intermunicipal e/ou interestadual de passageiros, e 20 UF não implementaram a quarentena para toda a população (Tabela 1).

As primeiras medidas de distanciamento social implementadas no Brasil ocorreram no Distrito Federal, no dia 11 de março de 2020. Nas demais UF, a maioria das medidas foi implementada na segunda quinzena de março, no período de 13 a 28 de março de 2020. O tempo em dias entre a adoção da primeira medida e a paralisação econômica (plena ou parcial) em cada UF é apresentado na Tabela 1. Mato Grosso do Sul, Santa Catarina e Rio Grande do Sul foram as UF que adotaram esses grupos de medidas em menor espaço de tempo, com um a dois dias de diferença. No outro extremo, no Pará, o tempo entre a implementação da primeira medida e da paralisação econômica foi de 50 dias. Em $74 \%$ das UF, o tempo entre a implementação da primeira medida e a paralisação econômica (plena ou parcial) foi igual ou inferir a uma semana.

O momento epidemiológico da implementação das categorias de medidas de distanciamento social teve pouca variação entre as UF brasileiras, uma vez que a maioria implementou todas elas, com exceção da categoria quarentena da população, entre o primeiro e o décimo caso de COVID-19. Para as categorias suspensão de eventos, suspensão de aulas e quarentena de grupos de risco, observou-se que algumas UF, principalmente das regiões Norte e Nordeste do país, implementaram esse conjunto de medidas antes da notificação do primeiro caso da doença. Somente as UF da Paraíba, do Espírito Santo e do Maranhão adotaram alguma categoria de medidas após o quinquagésimo caso 
Tabela 1

Data da implementação das categorias de medidas de distanciamento social e tempo em dias entre a implementação da primeira medida e a categoria paralisação econômica (plena ou parcial) por Unidade da Federação (UF), Brasil, 2020.

\begin{tabular}{|c|c|c|c|c|c|c|c|c|}
\hline UF & $\begin{array}{l}\text { Suspensão } \\
\text { de eventos }\end{array}$ & $\begin{array}{l}\text { Suspensão } \\
\text { de aulas }\end{array}$ & $\begin{array}{c}\text { Quarentena } \\
\text { de grupos } \\
\text { de risco }\end{array}$ & $\begin{array}{l}\text { Paralisação } \\
\text { econômica } \\
\text { plena }\end{array}$ & $\begin{array}{l}\text { Paralisação } \\
\text { econômica } \\
\text { parcial }\end{array}$ & $\begin{array}{l}\text { Restrição do } \\
\text { transporte }\end{array}$ & $\begin{array}{c}\text { Quarentena } \\
\text { da população }\end{array}$ & $\begin{array}{l}\text { Tempo entre } \\
\text { 1a medida } \\
\text { implementada } \\
\text { e paralisação } \\
\text { econômica } \\
\text { (em dias) }\end{array}$ \\
\hline MS & 20/março & 23/março & 20/março & NA & 21/março & NA & NA & 1 \\
\hline SC & 17/março & 19/março & $16 /$ março & NA & 18/março & 18/março & NA & 2 \\
\hline RS & 19/março & 19/março & 17/março & 19/março & NA & 19/março & 1/abril & 2 \\
\hline RO & 17/março & 17/março & 17/março & NA & 20/março & 20/março & 20/março & 3 \\
\hline SE & 17/março & 17/março & 17/março & NA & 20/março & 23/março & NA & 3 \\
\hline AP & 20/março & 18/março & 17/março & 20/março & NA & 23/março & 3/abril & 3 \\
\hline RJ & 13/março & 13/março & 13/março & NA & 17/março & 17/março & NA & 4 \\
\hline$A C$ & 16/março & 18/março & 20/março & 20/março & NA & 20/março & NA & 4 \\
\hline CE & 16/março & 19/março & 16/março & 20/março & NA & 23/março & NA & 4 \\
\hline MA & 16/março & 17/março & 22/março & 21/março & NA & 21/março & 20/maio & 5 \\
\hline PR & 16/março & 20/março & 16/março & NA & 21/março & 20/março & NA & 5 \\
\hline PB & 17/março & 19/março & 19/março & NA & 22/março & 20/maio & NA & 5 \\
\hline MG & 19/março & 18/março & 17/março & NA & 22/março & 23/março & NA & 5 \\
\hline TO & 21/março & 16/março & 21/março & NA & 21/março & NA & NA & 5 \\
\hline GO & 13/março & 18/março & 14/março & 20/março & NA & 20/março & NA & 6 \\
\hline ES & 17/março & 23/março & 18/março & NA & 23/março & NA & 25/maio & 6 \\
\hline PI & 16/março & 16/março & 18/março & NA & 23/março & 6/abril & NA & 7 \\
\hline RR & 16/março & 17/março & 23/março & NA & 23/março & 23/março & NA & 7 \\
\hline MT & 16/março & 23/março & 18/março & NA & 23/março & 23/março & NA & 7 \\
\hline AM & 17/março & 19/março & 16/março & NA & 23/março & 19/março & NA & 7 \\
\hline$A L$ & 13/março & 23/março & 16/março & 21/março & NA & 23/março & NA & 8 \\
\hline PE & 14/março & 18/março & 17/março & NA & 22/março & 22/março & NA & 8 \\
\hline SP & 13/março & 23/março & 17/março & NA & 24/março & NA & 24/março & 11 \\
\hline BA & 19/março & 19/março & 17/março & NA & 28/março & 20/março & NA & 11 \\
\hline DF & 11/março & 11/março & 17/março & NA & 23/março & NA & NA & 12 \\
\hline $\mathrm{RN}$ & 18/março & 18/março & 14/março & NA & 2/abril & NA & 2/abril & 19 \\
\hline PA & 16/março & 16/março & 16/março & NA & 5/maio & 23/março & NA & 50 \\
\hline
\end{tabular}

AC: Acre; AL: Alagoas; AM: Amazonas; AP: Amapá; BA: Bahia; CE: Ceará; DF: Distrito Federal: ES: Espírito Santo; GO: Goiás; MA: Maranhão; MG: Minas Gerais; MS: Mato Grosso do Sul; MT: Mato Grosso; NA: não aplicável; PA: Pará; PB: Paraíba; PE: Pernambuco; PI: Piauí; PR: Paraná; RJ: Rio de Janeiro; RN: Rio Grande do Norte; RO: Rondônia; RR: Roraima; SC: Santa Catarina; SE: Sergipe; SP: São Paulo; TO: Tocantins.

de COVID-19, sendo a categoria restrição do transporte na Paraíba e quarentena da população no Espírito Santo e Maranhão (Tabela 2).

Todas as sete UF que implementaram a categoria paralisação econômica plena o fizeram no período de até 13 dias após a notificação do primeiro caso de COVID-19. Amapá e Maranhão implementaram essa categoria quando contabilizavam um e dois casos de COVID-19, respectivamente, e Ceará, 68 casos da doença. Nas UF que adotaram essa categoria parcialmente, nas regiões Centro-Oeste, Sul e Sudeste, Mato Grosso o fez quando haviam sido notificados seis casos da doença enquanto São Paulo já contabilizava 810 casos. Nas regiões Norte e Nordeste, Roraima e Paraíba tinham um caso, Bahia 127 casos e Pará 4.756 casos de COVID-19 notificados no momento da implementação da medida (Figura 1). 


\section{Tabela 2}

Momento epidemiológico em que cada Unidade da Federação (UF) brasileira implementou as categorias de medidas de distanciamento social segundo número de casos confirmados de COVID-19. Brasil, 2020.

\begin{tabular}{|c|c|c|c|c|}
\hline \multirow[t]{2}{*}{ Categorias } & \multicolumn{4}{|c|}{ Momento epidemiológico da implementação das medidas de distanciamento social } \\
\hline & $\begin{array}{c}\text { Precoce } \\
\text { (antes do 1o caso) }\end{array}$ & $\begin{array}{c}\text { Intermediário I } \\
\text { (caso } 1 \text { ao caso 10) }\end{array}$ & $\begin{array}{c}\text { Intermediário II } \\
\text { (caso } 11 \text { ao caso 50) }\end{array}$ & $\begin{array}{c}\text { Tardio } \\
\text { (a partir do caso 50) }\end{array}$ \\
\hline Suspensão de eventos & $\begin{array}{c}A C, M A, M T, P A, P B, P I, \\
R O, R R\end{array}$ & $\begin{array}{l}\text { AL, AM, AP, CE, DF, GO, MS, } \\
\text { PE, PR, RJ, RN, RS, SC, SE, TO }\end{array}$ & $B A, E S, M G, S P$ & - \\
\hline Suspensão de aulas & $\begin{array}{c}A P, M A, P A, P I, R O, R R, \\
\text { TO }\end{array}$ & $\begin{array}{c}\text { AC, AM, CE, DF, GO, MG, MS, } \\
M T, P B, P E, P R, R J, R N, R S, \\
\text { SC, SE }\end{array}$ & $A L, B A, E S, S P$ & - \\
\hline Quarentena de grupos de risco & $A P, M T, P A, P I, R O$ & $\begin{array}{c}A C, A L, A M, C E, D F, G O, M A \\
M G, M S, P B, P E, P R, R J, R N \\
\text { RR, RS, SC, SE, TO }\end{array}$ & $\mathrm{BA}, \mathrm{ES}, \mathrm{SP}$ & - \\
\hline Paralisação econômica plena & - & $A C, A P, C E, G O, M A, R S$ & $\mathrm{AL}$ & - \\
\hline Paralisação econômica parcial & - & $\begin{array}{c}\text { AM, MS, MT, PB, PE, PI, PR, } \\
\text { RO, RR, SC, SE, TO }\end{array}$ & $\begin{array}{c}\text { BA, DF, ES, MG, PA, RJ, } \\
\text { RN, SP }\end{array}$ & - \\
\hline Restrição do transporte * & - & $\begin{array}{l}\text { AC, AM, AP, CE, GO, MA, MT, } \\
P A, P E, P R, R O, R R, R S, S C, S E\end{array}$ & $\mathrm{AL}, \mathrm{BA}, \mathrm{MG}, \mathrm{PI}, \mathrm{RJ}$ & PB \\
\hline Quarentena da população * & - & $\mathrm{RO}$ & $A P, R N, R S, S P$ & ES, MA \\
\hline
\end{tabular}

AC: Acre; AL: Alagoas; AM: Amazonas; AP: Amapá; BA: Bahia; CE: Ceará; DF: Distrito Federal: ES: Espírito Santo; GO: Goiás; MA: Maranhão; MG: Minas Gerais; MS: Mato Grosso do Sul; MT: Mato Grosso; PA: Pará; PB: Paraíba; PE: Pernambuco; PI: Piauí; PR: Paraná; RJ: Rio de Janeiro; RN: Rio Grande do Norte; RO: Rondônia; RR: Roraima; SC: Santa Catarina; SE: Sergipe; SP: São Paulo; TO: Tocantins.

* Não foram apresentadas as UF que não implementaram essa categoria de medidas de distanciamento social.

O número de dias anteriores ou posteriores à notificação do primeiro óbito por COVID-19 em cada UF, em relação à data da implementação das categorias de medidas de distanciamento social, é apresentado na Tabela 3. A maioria das UF implementou as medidas antes do primeiro óbito, com destaque para Tocantins, que iniciou a sua implementação trinta dias antes do primeiro óbito notificado. Em relação à implementação da categoria paralisação econômica parcial, Pará, Rio Grande do Norte e São Paulo foram as únicas UF que adotaram essa medida após o primeiro óbito, com intervalo de 34, 5 e 7 dias, respectivamente.

\section{Discussão}

No Brasil, o Supremo Tribunal Federal atribuiu aos estados, Distrito Federal e municípios a competência por decidir implementar as medidas de distanciamento social para mitigação e supressão da COVID-19 22. Nesse sentido, foram poucas as medidas implementadas no nível federal, limitandose à restrição da entrada de estrangeiros no país 25 e à determinação para que pessoas com mais de sessenta anos de idade observem o distanciamento social, restringindo seus deslocamentos para a realização de atividades estritamente necessárias 26. Além dessas medidas, o Ministério da Saúde publicou um plano de ação no dia 14 de março de 2020 recomendando que as Secretarias de Saúde dos municípios, estados e Distrito Federal avaliassem a adoção de medidas não farmacológicas para o controle da COVID-19, de acordo com as fases de transmissão da doença 27.

Como em outros países, as UF brasileiras implementaram não somente uma medida isolada, mas um conjunto de medidas de distanciamento social que, segundo evidências, apresentam maior efetividade na mitigação e supressão da COVID-19 principalmente quando associado ao isolamento dos casos e à quarentena dos contatos 11,28,29. Outro achado importante é que a implementação progressiva de medidas de diferentes categorias ocorreu em um curto espaço de tempo, culminando, em sua 
Figura 1

Momento epidemiológico da implementação das categorias paralisação econômica plena, paralisação econômica parcial para as Unidades da Federação (UF) das regiões Sul, Sudeste e Centro-oeste e paralisação econômica parcial para as UF das regiões Norte e Nordeste , a partir do primeiro caso confirmado de COVID-19. Brasil, 2020.

1a) Paralisação econômica plena

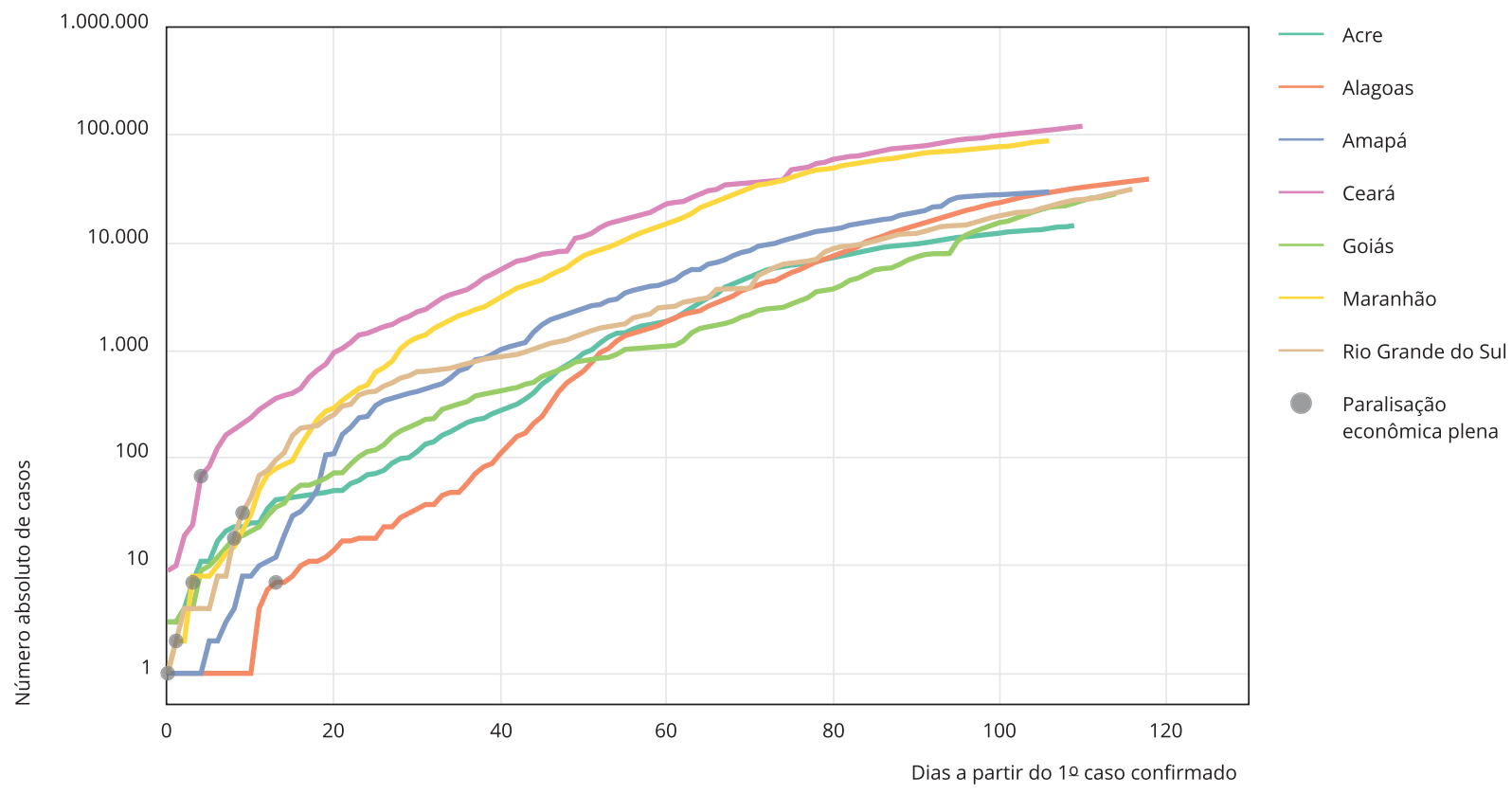

1b) Paralisação econômica parcial para as UF das regiões Sul, Sudeste e Centro-oeste
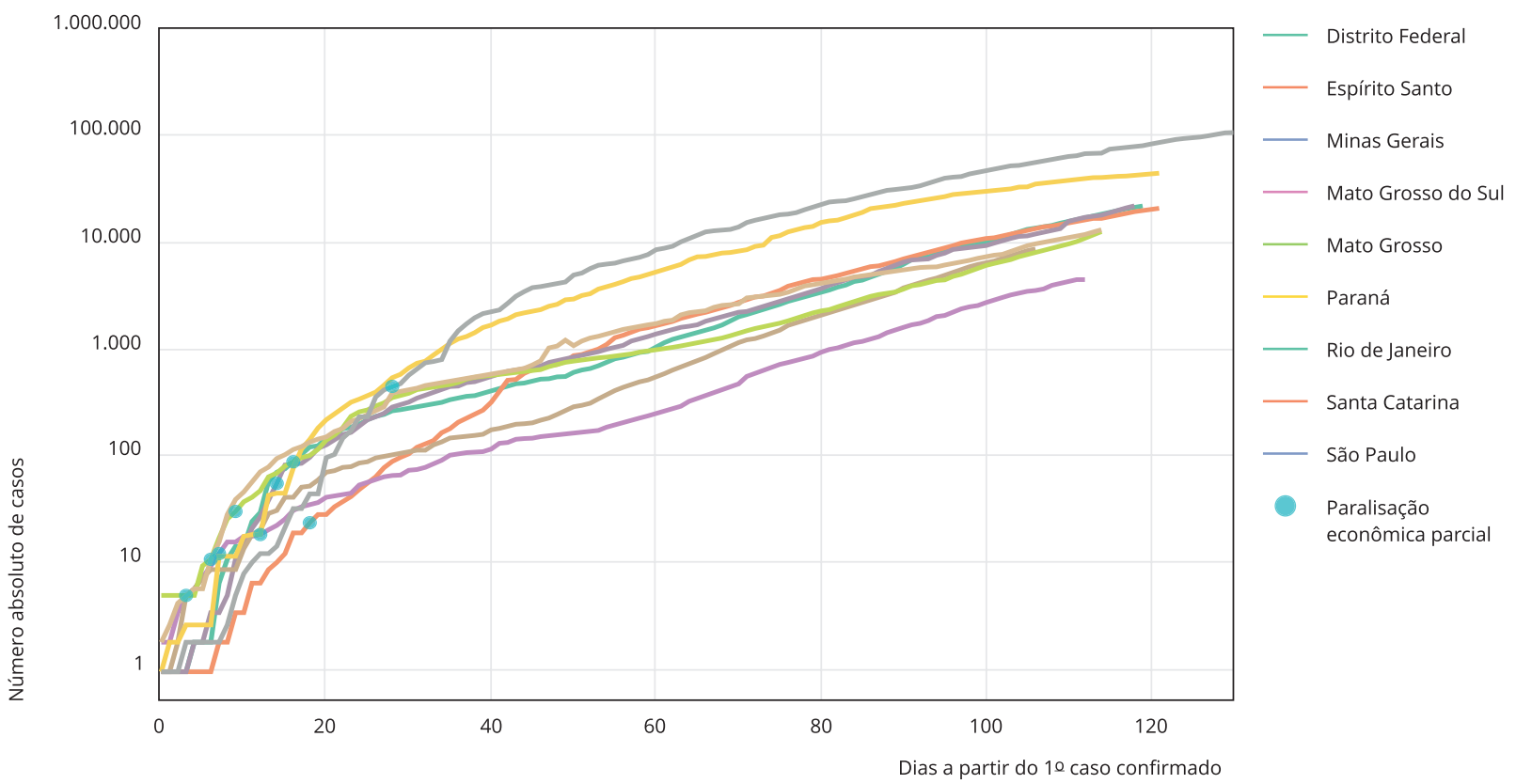

(continua) 
1c) Paralisação econômica parcial para as UF das regiões Norte e Nordeste

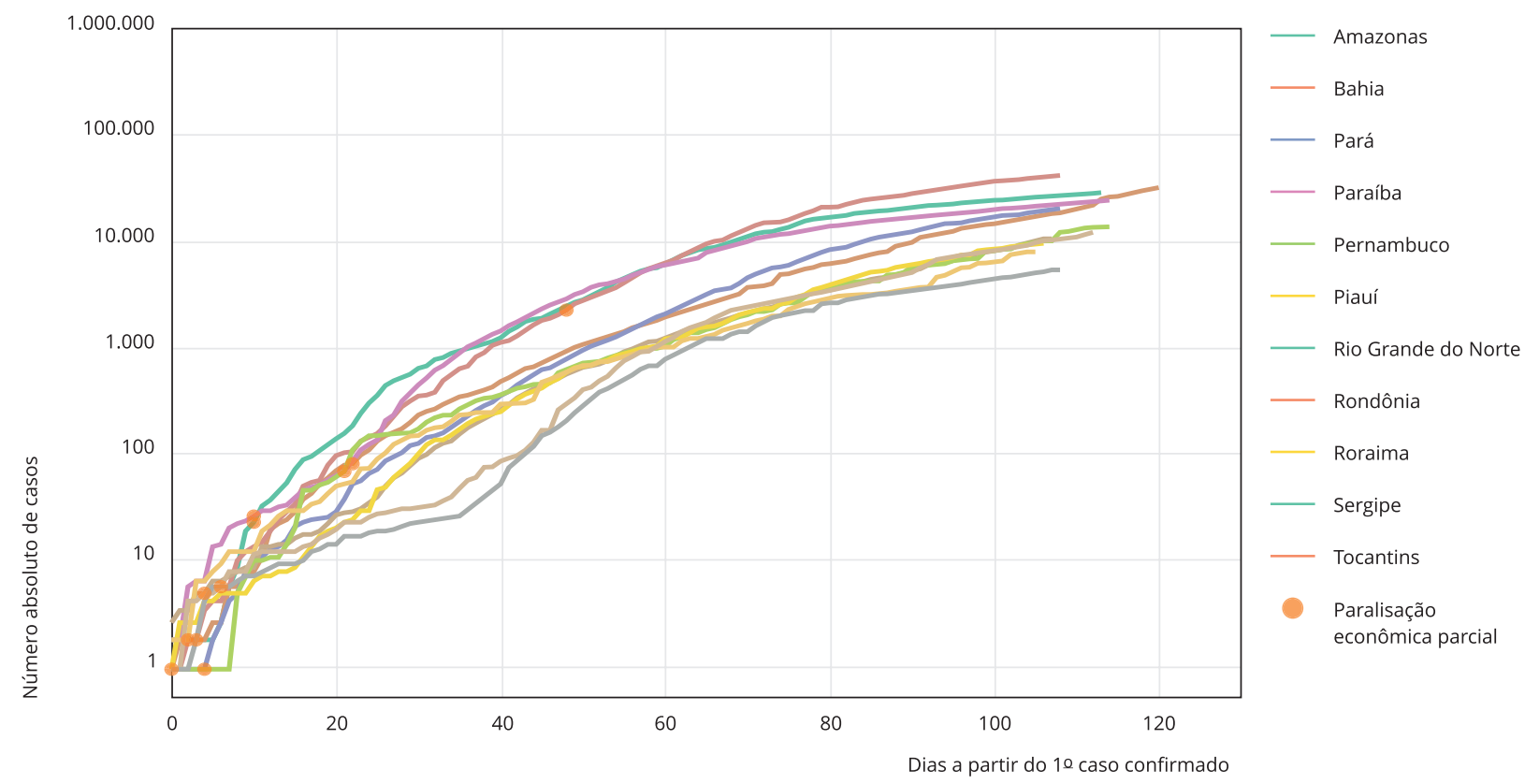

maioria, na paralisação plena ou parcial das atividades econômicas não essenciais, seguindo sequência usualmente observada em outros países que também adotaram essas medidas para o enfretamento da COVID-19 30

O Distrito Federal foi a primeira UF do país a implementar uma série de medidas de distanciamento social, exatamente no dia em que a OMS declarou a pandemia de COVID-19 1. A partir dessa data, todas as UF iniciaram a adoção das medidas que se concentraram, em sua maioria, na segunda quinzena de março, antes mesmo de completar um mês da confirmação do primeiro caso da doença no país 19. Esses achados corroboram os do grupo de pesquisa da Universidade de Oxford (The Oxford COVID-19 Government Response Tracker - OxCGRT) que, ao realizar o levantamento e monitoramento das medidas de distanciamento social em mais de 160 países, identificou que, em geral, as respostas dos governos em relação ao enfrentamento da COVID-19 têm se tornado mais fortes ao longo da epidemia, com aumento expressivo observado no mês de março, apesar das variações entre os países 31 .

Na maioria das UF brasileiras, a implementação de grande parte das medidas de distanciamento social ocorreu antes da notificação do décimo caso e do primeiro óbito pela doença, o que foi precoce em relação aos achados de Summan \& Nandi ${ }^{29}$. Ao avaliar o momento da implementação das medidas de distanciamento social em vários países do mundo, eles identificaram que a suspensão de aulas ocorreu, em média, 13 dias após o primeiro caso detectado de COVID-19, seguido de restrições de viagens aéreas internacionais com média de 18 dias e lockdowns nacionais com média de 21 dias após o primeiro caso da doença 29. Summan \& Nandi 29 também identificaram que países mais pobres, com maior número de notificações após duas semanas do primeiro caso, sistemas menos democráticos e menos populosos, e com população mais jovem e menos densa implementaram essas medidas mais precocemente, ao passo que países com renda mais alta, maiores populações e mais preparados em relação à resposta do sistema de saúde adotaram as medidas mais tardiamente em relação à ocorrência de casos de COVID-19 localmente 29. 
Tabela 3

Número de dias anteriores (-) ou posteriores (+) a implementação de cada categoria de medidas de distanciamento social a partir do 1 o óbito por COVID-19 em cada Unidade da Federação (UF). Brasil, 2020.

\begin{tabular}{|c|c|c|c|c|c|c|c|c|}
\hline UF & $\begin{array}{l}\text { Suspensão } \\
\text { de eventos }\end{array}$ & $\begin{array}{l}\text { Suspensão } \\
\text { de aulas }\end{array}$ & $\begin{array}{c}\text { Quarentena } \\
\text { de grupos } \\
\text { de risco }\end{array}$ & $\begin{array}{c}\text { Paralisação } \\
\text { econômica } \\
\text { plena }\end{array}$ & $\begin{array}{c}\text { Paralisação } \\
\text { econômica } \\
\text { parcial }\end{array}$ & $\begin{array}{l}\text { Restrição de } \\
\text { transporte }\end{array}$ & $\begin{array}{c}\text { Quarentena } \\
\text { da população }\end{array}$ & $\begin{array}{c}\text { Data do } 10 \\
\text { óbito }\end{array}$ \\
\hline TO & -25 & -30 & -25 & & -25 & & & 15/março \\
\hline$A C$ & -21 & -19 & -17 & -17 & & -17 & & 6/abril \\
\hline DF & -18 & -18 & -12 & & -6 & & & 29/março \\
\hline $\mathrm{RR}$ & -18 & -17 & -11 & & -11 & -11 & & 3/abril \\
\hline MT & -18 & -11 & -16 & & -11 & -11 & & 3/abril \\
\hline $\mathrm{AL}$ & -18 & -8 & -15 & -10 & & -8 & & 31/março \\
\hline SE & -16 & -16 & -16 & & -13 & -10 & & 2/abril \\
\hline PA & -16 & -16 & -16 & & 34 & -9 & & 1/abril \\
\hline ES & -16 & -10 & -15 & & -10 & -10 & 53 & 2/abril \\
\hline AP & -15 & -17 & -18 & -15 & & -12 & -1 & 4/abril \\
\hline PB & -14 & -12 & -12 & & -9 & 50 & & 31/março \\
\hline RO & -13 & -13 & -13 & & -10 & -10 & -10 & 30/março \\
\hline MA & -13 & -12 & -7 & -8 & & -8 & 52 & 29/março \\
\hline GO & -13 & -8 & -12 & -6 & & -6 & & 26/março \\
\hline MS & -11 & -8 & -11 & & -10 & & & 31/março \\
\hline MG & -11 & -12 & -13 & & -8 & -7 & & 30/março \\
\hline $\mathrm{PI}$ & -11 & -11 & -9 & & -4 & 10 & & 27/março \\
\hline PR & -11 & -7 & -11 & & -6 & -7 & & 27/março \\
\hline PE & -11 & -7 & -8 & & -3 & -3 & & 25/março \\
\hline $\mathrm{RN}$ & -10 & -10 & -14 & & 5 & & 5 & 28/março \\
\hline BA & -10 & -10 & -12 & & -1 & -9 & & 29/março \\
\hline CE & -10 & -7 & -10 & -6 & & -3 & & 26/março \\
\hline SC & -9 & -7 & -10 & & -8 & -8 & & 26/março \\
\hline AM & -7 & -5 & -8 & & -1 & -5 & & 24/março \\
\hline $\mathrm{RJ}$ & -6 & -6 & -6 & & -2 & -2 & & 19/março \\
\hline RS & -6 & -6 & -8 & -6 & & -6 & 7 & 25/março \\
\hline $\mathrm{SP}$ & -4 & 6 & 0 & & 7 & & 7 & $17 /$ março \\
\hline
\end{tabular}

AC: Acre; AL: Alagoas; AM: Amazonas; AP: Amapá; BA: Bahia; CE: Ceará; DF: Distrito Federal: ES: Espírito Santo; GO: Goiás; MA: Maranhão; MG: Minas Gerais; MS: Mato Grosso do Sul; MT: Mato Grosso; NA: não aplicável; PA: Pará; PB: Paraíba; PE: Pernambuco; PI: Piauí; PR: Paraná; RJ: Rio de Janeiro; RN: Rio Grande do Norte; RO: Rondônia; RR: Roraima; SC: Santa Catarina; SE: Sergipe; SP: São Paulo; TO: Tocantins.

Nota: a gradação das cores estabelece o período de implementação de cada categoria, sendo o mais intenso referente ao período de 21 a 30 dias anteriores do primeiro óbito, seguido por 20 a 11 dias anteriores, 10 a 1 dia anterior, 0 a 10 dias posteriores e acima de 10 dias posteriores ao primeiro óbito.

Evidências sugerem que a adoção precoce das medidas de distanciamento social é fundamental para frear a transmissão da doença e, consequentemente, achatar a curva de casos e reduzir a demanda dos serviços de saúde 3,14,32,33,34. Estudo que simulou a epidemia de COVID-19 nas cidades da China Continental estimou que, se uma série de intervenções não farmacológicas, incluindo o distanciamento social, tivesse sido implementada em uma, duas ou três semanas antes do início da epidemia, o número de casos de COVID-19 poderia ter sido reduzido em 66\%, 86\% e 95\%, respectivamente, além de restringir significativamente o número de áreas afetadas 35. Nos Estados Unidos, estudo realizado entre março e abril de 2020 concluiu que a adoção de medidas de distanciamento social impostas pelo governo reduziu a taxa de crescimento diário em 5,4 pontos percentuais após 1 a 5 dias, 6,8 após 6 a 10 dias, 8,2 após 11 a 15 dias e 9,1 após 16 a 20 dias 34 . 
No Brasil, estudo de série temporal de óbitos pela COVID-19 realizado no Estado de São Paulo demonstrou que as estratégias de distanciamento social implementadas no estado entre os dias 16 e 22 de março de 2020 resultaram em decréscimo substancial do número de infecções pela doença, sendo a redução mais evidente na cidade de São Paulo entre os dias 5 e 20 de abril. $O$ autor justifica que a diferença de tempo entre a implementação das medidas e a redução do número de óbitos foi cerca de 17 dias, tempo equivalente ao início dos sintomas e ao óbito por COVID-19 36. Esses achados corroboram aqueles relatados em outro estudo que, ao avaliar o impacto das intervenções não farmacológicas nas cidades de São Paulo e Rio de Janeiro, identificou, por meio de modelos de transmissão orientados pela mobilidade, que a taxa de reprodução efetiva (Rt) do SARS-CoV-2 ao longo do tempo nessas cidades chegou a ficar abaixo de 1 logo após a implementação das intervenções não farmacológicas, mas aumentou para valores entre 1 e 1,3 após a flexibilização dessas medidas nesses locais 37.

Juntamente com o momento da implementação, a rigidez e duração das medidas também parecem ser fundamentais para o sucesso dessa intervenção 11. O OxCGRT identificou que, até o dia 30 de maio de 2020, a maioria dos mais de 160 países monitorados por esse grupo apresentava o índice de rigidez das medidas de distanciamento acima de 70 (em uma escala de 0-100, sendo o 100 mais rígido), sendo o índice do Brasil igual a 81,02 30. Esse índice também está sendo monitorado nos estados brasileiros e suas capitais pelo Instituto de Pesquisa Econômica Aplicada (IPEA) 38, que observou o aumento contínuo do grau de rigidez das medidas nos estados brasileiros e capitais até os dias 23 e 24 de março de 2020, apesar de alguns estados adotarem políticas substancialmente mais rigorosas que outros 39 , e já se observa uma diminuição no rigor das medidas desde o início de junho, permanecendo as variações entre os diferentes estados e capitais 40 .

A duração do isolamento deve ser longo o suficiente para que os efeitos epidemiológicos desejáveis sejam alcançados, causando o mínimo de danos sociais e econômicos 3 e evitando o desgaste e a perda da adesão por parte da população, como já se tem observado em muitas UF brasileiras 40 . Do ponto de vista socioeconômico, estudo de revisão já aponta que o distanciamento social, o autoisolamento e as restrições de viagens têm resultado em desemprego em muitos setores econômicos, sobrecarga de trabalho em outros, além do aumento de casos de violência doméstica (física, emocional e sexual) 18. Outro estudo também aponta que a epidemia de COVID-19 está causando problemas de saúde adicionais, como estresse, ansiedade, sintomas depressivos, insônia, entre outras doenças mentais, apesar de reconhecer que estudos relacionados à saúde mental ainda são escassos 41 . Esses achados têm levado alguns pesquisadores a optar pela utilização do termo "distanciamento físico" em vez de "distanciamento social" para reforçar a necessidade de estarmos conectados socialmente, mantendo-nos, ao mesmo tempo, distantes fisicamente.

Deve-se acrescentar que a implementação muito precoce das medidas de distanciamento social pode resultar também em uma flexibilização precoce dessas mesmas medidas, em momentos em que epidemiologicamente se observa aumento crescente de casos e óbitos. O quanto e de que maneira a precocidade da implementação das medidas de distanciamento social nas UF brasileiras impactou na progressão da epidemia mas também em possíveis flexibilizações prematuras em várias UF é algo que deve ser avaliado em estudos específicos para essa finalidade.

Tem sido proposta na literatura internacional a implementação de estratégias intermitentes, com periodicidades variadas, incluindo restrição e flexibilização ao longo do tempo, considerando indicadores epidemiológicos. Essa abordagem está sendo considerada para o futuro imediato, até que uma vacina segura e efetiva esteja disponível, uma vez que se acredita que, em virtude do grande número de suscetíveis, deva ocorrer uma segunda onda de infecções por SARS-CoV-2 42.

O presente estudo tem limitações que devem ser consideradas. Por ser um estudo descritivo, não tem o potencial de verificar o efeito de medidas de distanciamento na evolução dos casos e óbitos de COVID-19, mas levanta hipóteses que podem ser validadas em estudos posteriores. Acrescenta-se ainda que foi realizado o levantamento da legislação das UF, e não do nível municipal. Conforme a determinação do Supremo Tribunal Federal de 15 de abril de 2020, os municípios têm autonomia para definir e decretar recomendações diferentes das adotadas nas UF 22. Outra limitação é que foram identificadas as medidas de distanciamento determinadas ou recomendadas pela legislação da UF, mas a adesão a essas medidas não pôde ser avaliada em face do desenho do escopo do estudo.

Em conclusão, observamos, no presente estudo, que todas as UF brasileiras implementaram as medidas de distanciamento social de forma abrangente, precoce e em um curto espaço de tempo, 
com vistas a mitigar o impacto da COVID-19. No entanto, o impacto dessa implementação, tanto na evolução epidemiológica da doença quanto em uma possível flexibilização precoce em momentos epidemiológicos inadequados, ainda não está claro. Ressalta-se, portanto, a necessidade de um monitoramento constante e atento da evolução dos casos dessa doença nas UF que já estão flexibilizando as medidas, para que os esforços alcançados na mitigação da COVID-19 não venham a se perder com essa liberação. Ressalta-se a necessidade de os gestores se basearem em evidências para a tomada de decisão em relação a quando implementar, flexibilizar ou reintroduzir as medidas de distanciamento social em cada UF, assim como a colaboração da sociedade no enfrentamento dessa doença, no sentido de seguir as recomendações em níveis individual, ambiental e comunitário.

\section{Colaboradores}

L. L. S. Silva, A. F. R. Lima e D. A. Polli contribuíram com a coleta e análise dos dados, além da redação e revisão da versão final do manuscrito. P. F. S. Razia e L. F. A. Pavão contribuíram com a coleta dos dados, além da revisão da versão final do manuscrito. M. A. F. H. Cavalcanti e C. M. Toscano contribuíram com o desenvolvimento intelectual do projeto, validação da metodologia, redação e revisão da versão final do manuscrito.

\section{Informações adicionais}

ORCID: Lara Lívia Santos da Silva (0000-00028948-7884); Alex Felipe Rodrigues Lima (00000003-2591-1452); Démerson André Polli (00000002-5904-2315); Paulo Fellipe Silvério Razia (0000-0001-8406-7453); Luis Felipe Alvim Pavão (0000-0001-9265-456X); Marco Antônio Freitas de Hollanda Cavalcanti (0000-0002-1159-0785); Cristiana Maria Toscano (0000-0002-9453-2643).

\section{Referências}

1. World Health Organization. Coronavirus disease 2019 (COVID-19). Situation report - 51. https://www.who.int/docs/default-source/ coronaviruse/situation-reports/20200311sitrep-51-covid-19.pdf?sfvrsn=1ba62e57_10 (acessado em 11/Jun/2020).

2. World Health Organization. Coronavirus disease (COVID-19). Situation Report - 142. https://www.who.int/docs/default-source/ coronaviruse/situation-reports/20200610covid-19-sitrep-142.pdf?sfvrsn=180898cd_6 (acessado em 11/Jun/2020).

3. Cowling BJ, Aiello AE. Public health measures to slow community spread of coronavirus disease 2019. J Infect Dis 2020; 221:1749-51.

4. Anderson RM, Heesterbeek H, Klinkenberg D, Hollingsworth TD. How will country-based mitigation measures influence the course of the COVID-19 epidemic? Lancet 2020; 395:931-4.

5. World Health Organization. Overview of public health and social measures in the context of COVID-19 (Interim guidance). https:// www.who.int/publications/i/item/overviewof-public-health-and-social-measures-inthe-context-of-covid-19 (acessado em 11/ Jun/2020)

6. Markel H, Lipman HB, Navarro JA, Sloan A, Michalsen JR, Stern AM, et al. Nonpharmaceutical interventions implemented by US cities during the 1918-1919 influenza pandemic. JAMA 2007; 298:644-54.

7. Bell DM; World Health Organization Working Group on Prevention of International and Community Transmission of SARS. Public health interventions and SARS spread, 2003. Emerg Infect Dis 2004; 10:1900-6. 
8. Fong MW, Gao H, Wong JY, Xiao J, Shiu EYC, Ryu S, et al. Nonpharmaceutical measures for pandemic influenza in nonhealthcare settingssocial distancing measures. Emerg Infect Dis 2020; 26:976-84.

9. European Center for Disease Prevention and Control. Coronavirus disease 2019 (COVID-19) pandemic: increased transmission in the EU/EEA and the UK - seventh update. https://www.ecdc.europa.eu/sites/de fault/files/documents/RRA-seventh-updateOutbreak-of-coronavirus-disease-COVID-19. pdf (acessado em 11/Jun/2020).

10. Government of Canada. Community-based measures to mitigate the spread of coronavirus disease (COVID-19) in Canada. https:// www.canada.ca/en/public-health/services/ diseases/2019-novel-coronavirus-infection/ health-professionals/public-health-measuresmitigate-covid-19.html (acessado em 11/ Jun/2020).

11. Ferguson NM, Laydon D, Nedjati-Gilani G, Imai N, Ainslie K, Baguelin M, et al. Impact of non-pharmaceutical interventions (NPIs) to reduce COVID-19 mortality and healthcare demand. https://www.imperial.ac.uk/media/ imperial-college/medicine/sph/ide/gida-fel lowships/Imperial-College-COVID19-NPImodelling-16-03-2020.pdf (acessado em 10/ Jun/2020).

12. Lau H, Khosrawipour V, Kocbach P, Mikolajczyk A, Schubert J, Bania J, et al. The positive impact of lockdown in Wuhan on containing the COVID-19 outbreak in China. J Travel Med 2020; 2020; 27:taaa037.

13. Cohen J, Kupferschmidt K. Countries test tactics in 'war' against COVID-19. Science 2020; 367:1287-8.

14. Nussbaumer-Streit B, Mayr V, Dobrescu AI, Chapman A, Persad E, Klerings I, et al. Quarantine alone or in combination with other public health measures to control COVID-19: a rapid review. Cochrane Database Syst Rev 2020; 4:CD013574.

15. Wu P, Tsang TK, Wong JY, Ng TWY, Ho F, Gao $\mathrm{H}$, et al. Suppressing COVID-19 transmission in Hong Kong: an observational study of the first four months. Research Square 2020; 9 jun. https://www.researchsquare.com/article/rs$34047 / \mathrm{v} 1$.

16. Figueiredo AM, Codina AD, Figueiredo DCMM, Saez M, León AC. Impact of lockdown on COVID-19 incidence and mortality in China: an interrupted time series study. Bull World Health Organ 2020; 6 abr. http://dx.doi. org/10.2471/BLT.20.256701.

17. Oliveira C. Does "staying at home" save lives? An estimation of the impacts of social isolation in the registered cases and deaths by Covid-19 in Brazil. SSRN 2020; 13 mai. https://papers. ssrn.com/sol3/papers.cfm?abstract_id =359 3947.
18. Nicola M, Alsafi Z, Sohrabi C, Kerwan A, AlJabir A, Iosifidis C, et al. The socio-economic implications of the coronavirus and COVID-19 pandemic: a review. Int J Surg 2020; 78:18593.

19. Ministério da Saúde. Boletim Epidemiológico Especial - 14. COE-COVID19. https://por talarquivos.saude.gov.br/images/pdf/2020/ April/27/2020-04-27-18-05h-BEE14-Bole tim-do-COE.pdf (acessado em 11/Mai/2020).

20. Ministério da Saúde. Brasil registra 2.915 casos confirmados de coronavírus e 77 mortes. https://www.saude.gov.br/noticias/agenciasaude/46610-brasil-registra-2-915-casosconfirmados-de-coronavirus-e-77-mortes (acessado em 11/Mai/2020).

21. Ministério da Saúde. Painel Coronavírus. https://covid.saude.gov.br/ (acessado em 11/ Mai/2020).

22. Supremo Tribunal Federal. STF reconhece competência concorrente de estados, DF, municípios e União no combate à Covid-19. http://www.stf.jus.br/portal/cms/verNoticia Detalhe.asp?idConteudo $=441447$ (acessado em 11/Jun/2020).

23. Ministério da Saúde. Boletim Epidemiológico - 04. https://www.saude.gov.br/images/ pdf/2020/marco/04/2020-03-02-Boletim-Epi demiol--gico-04-corrigido.pdf (acessado em 11/Mai/2020).

24. Brasil.IO. Metodologia de coleta dos dados. https://docs.google.com/document/d/1escum cbjS8inzAKvuXOQocMcQ8ZCqbyHU5X5h FrPpn4/edit\# (acessado em 13/Mai/2020).

25. Brasil. Portal da Legislação: Legislação COVID-19. http://www4.planalto.gov.br/ legislacao/portal-legis/legislacao-covid-19 (acessado em 11/Jun/2020).

26. Ministério da Saúde. Portaria no 454 de 20 de março de 2020. Declara, em todo o território nacional, o estado de transmissão comunitária do coronavírus (covid-19). Diário Oficial da União 2020; 20 mar.

27. Ministério da Saúde. Boletim Epidemiológico no 06 do Centro de Operações de Emergências em Saúde Pública (COE COVID-19). https:// www.saude.gov.br/images/pdf/2020/mar co/24/03--ERRATA---Boletim-Epidemiologi co-05.pdf (acessado em 11/Jun/2020).

28. Cowling BJ, Ali ST, Ng TWY, Tsang TK, Li JCM, Fong MW, et al. Impact assessment of non-pharmaceutical interventions against coronavirus disease 2019 and influenza in Hong Kong: an observational study. Lancet Public Health 2020; 5:e279-88.

29. Summan A, Nandi A. Timing of non-pharmaceutical interventions to mitigate COVID-19 transmission and their effects on mobility: a cross-country analysis. medRxiv 2020; 13 mai. https://www.medrxiv.org/content/10.1101/2 020.05.09.20096420v1. 
30. Hale T, Webster S, Petherick A, Phillips T, Kira B. Oxford COVID-19 Government Response Tracker, Blavatnik School of Government; 2020. https://www.bsg.ox.ac.uk/research/ research-projects/coronavirus-governmentresponse-tracker (acessado em 15/Jun/2020).

31. Hale T, Webster S, Petherick A, Phillips T, Kira B. Variation in government responses to COVID-19 (version 6.0). Blavatnik School of Government working paper. https://www. bsg.ox.ac.uk/sites/default/files/2020-05/ BSG-WP-2020-032-v6.0.pdf (acessado em 15/ Jun/2020).

32. European Centre for Disease Prevention and Control. Considerations relating to social distancing measures in response to the COVID-19 epidemic. https://www.ecdc.europa.eu/en/ publications-data/considerations-relatingsocial-distancing-measures-response-covid19-second (acessado em 15/Jun/2020).

33. World Health Organization. Report of the WHO-China Joint Mission on Coronavirus Disease 2019 (COVID-19) https://www.who. int/docs/default-source/coronaviruse/whochina-joint-mission-on-covid-19-final-re port.pdf?sfvrsn=fce87f4e_2 (acessado em 15/ Jun/2020).

34. Courtemanche C, Garuccio J, Le A, Pinkston J, Yelowitz A. Strong social distancing measures in the United States reduced the COVID-19 growth rate. Health Aff (Millwood) 2020; 39:1237-46.

35. Lai S, Ruktanonchai NW, Zhou L, Prosper O, Luo W, Floyd JR, et al. Effect of non-pharmaceutical interventions for containing the COVID-19 outbreak: an observational and modelling study. medRxiv 2020; 13 mai. https://www.medrxiv.org/content/10.1101/2 020.03.03.20029843v3.
36. Cruz CHB. Social distancing in São Paulo State: demonstrating the reduction in cases using time series analysis of deaths due to COVID-19. Rev Bras Epidemiol 2020; 23:e200056.

37. Candido DS, Claro IM, Jesus JG, Souza WM, Moreira FRR, Dellicour S, et al. Evolution and epidemic spread of SARS-CoV-2 in Brazil. MedRxiv 2020; 23 jun. https://www.medrxiv. org/content/10.1101/2020.06.11.20128249v2.

38. Moraes RF. Índice de medidas legais de distanciamento social. http://tinyurl.com/ipeacoro navirus (acessado em 15/Jun/2020).

39. Moraes RF. Medidas legais de incentivo ao distanciamento social: comparação das políticas de governos estaduais e prefeituras das capitais no Brasil. Nota técnica 16. https://www.ipea. gov.br/portal/index.php?option=com_content $\&$ view $=$ article $\& i d=35462 \&$ Itemid $=4$ (acessado em 15/Jun/2020).

40. Moraes RF. COVID-19 e medidas legais de distanciamento social: isolamento social, gravidade da epidemia e análise do período de 25 de maio a 7 de junho de 2020 (boletim 5). Nota técnica 22. https://www.ipea.gov. $\mathrm{br} /$ portal/images/stories/PDFs/nota_tecni ca/200610_nt_dinte_n_22.pdf (acessado em 15/Jun/2020).

41. Torales J, O’Higgins M, Castaldelli-Maia JM, Ventriglio A. The outbreak of COVID-19 coronavirus and its impact on global mental health. Int J Soc Psychiatry 2020; 66:317-20.

42. Kissler SM, Tedijanto C, Goldstein E, Grad YH, Lipsitch M. Projecting the transmission dynamics of SARS-CoV-2 through the postpandemic period. Science 2020; 368:860-8. 


\section{Abstract}

Social distancing measures have been widely adopted to mitigate the COVID-19 pandemic. However, little is known about the timing of measures' implementation, scope, and duration in relation to their impact. The study aimed to describe the social distancing measures implemented by Brazil's states and the Federal District, including the types of measures and the timing of their implementation. This is a descriptive study of the measures type, chronological and epidemiological timing of the implementation, and scope. The survey of measures used searches in official websites of the government departments and each state's Government Register. The official number of COVID-19 cases and deaths were obtained from an official a data platform. We considered the following categories of social distancing measures: suspension of events, school closure, quarantine of risk groups, economic lockdown (partial or full), restrictions on transportation, and quarantine of the population. The implementation's timing considered both the chronological date and the epidemiological timing, based on the time since the 10th case or 1st death from COVID-19 in each state. All the states implemented distancing measures, mostly during the latter half of March 2020. Economic lockdown was implemented early, prior to the 10th case by $67 \%$ of the states and prior to the 1st death from COVID- 19 by $89 \%$ of the states. Early social distancing measures were widely implemented in Brazil, before or in the initial phase of the exponential growth curve of COVID-19 cases and deaths in the great majority of states.

COVID-19; Social Isolation; Epidemiology

\section{Resumen}

Medidas de distanciamiento social están siendo ampliamente adoptadas para mitigar la pandemia de la COVID-19. No obstante, poco se sabe en cuanto al momento de implementación, alcance y duración de la vigencia de las medidas en su impacto. El objetivo de este estudio fue caracterizar las medidas de distanciamiento social, implementadas por las Unidades de la Federación (UF) brasileñas, incluyendo el tipo de medida y el momento de su implementación. Se trata de un estudio descriptivo con caracterización del tipo, momento cronológico y epidemiológico de la implementación y alcance de las medidas. La obtención de las medidas se realizó a través de búsquedas en sitios oficiales de las Secretarías de Gobierno y Boletín Oficial de cada UF. Los números de casos y óbitos por COVID-19 se obtuvieron de una plataforma de información oficial. Consideramos las siguientes categorías de medidas de distanciamiento social: suspensión de eventos, suspensión de clases, cuarentena para grupos de riesgo, paralización económica (parcial o plena), restricción de transporte y cuarentena para la población. El momento de implementación consideró la fecha cronológica y también el momento epidemiológico, considerando el tiempo tras el 10o caso o ler óbito por COVID-19 en cada UF. Todas las UF implementaron medidas de distanciamiento, en su mayoría durante la segunda quincena de marzo de 2020. Se implementó la paralización económica precozmente, anterior al 10o caso por 67\% y anterior al 1er óbito por COVID-19 por $89 \%$ de las UF. Las medidas de distanciamiento social fueron ampliamente implementadas en Brasil, de manera precoz, antes o en la fase inicial de la curva de crecimiento exponencial de casos y óbitos por COVID-19 en la gran mayoría de las UF.

COVID-19; Aislamiento Social; Epidemiología
Recebido em 26/Jun/2020

Versão final reapresentada em 06/Jul/2020

Aprovado em 15/Jul/2020 